\title{
The Simultaneous Additive and Relative SysRem Algorithm
}

\author{
A. Ofir ${ }^{1}$ \\ 1 School of Physics and Astronomy, Tel Aviv University, Tel Aviv, Israel \\ [avivofir@wise.tau.ac.il]
}

\begin{abstract}
We present the SARS algorithm, which is a generalization of the popular SysRem detrending technique. This generalization allows including multiple external parameters in a simultaneous solution with the unknown effects. Using SARS allowed us to show that the magnitude-dependant systematic effect discovered by Mazeh et al. (2009) in the CoRoT data is probably caused by an additive -rather than relative- noise source. A post-processing scheme based on SARS performs well and indeed allows for the detection of new transit-like signals that were not previously detected.
\end{abstract}

\section{Introduction - The Problem}

Mazeh et al. 2009 (hereafter M09) discovered that there are significant magnitudedependant systematic effects in the CoRoT light curves (see Figure 1.). They developed a correction procedure for the new systematic effect, but it was is a purely phenomenological correction. Their correction had neither the ability to point to a particular cause for the effect, nor to justify the functional form (parabola) used at the heart of the correction, nor to properly correct bright stars.

\section{The SARS Solution}

We hypothesized that the underlying mechanism M09 were trying to correct for is a constant flux that is either added or subtracted from all the light curves. We therefore generalized (Ofir et al. 2010) the popular SysRem algorithm (Tamuz, Mazeh, \& Zucker 2005) to include both additive and relative terms in flux -therefore SARS="Simultaneous Additive and Relative SysRem". This was achieved by changing the SysRem noise model from $r_{i j}=R_{j} C_{R, i}$ To $r_{i j}=A_{j} x_{i j} C_{A, i}+R_{j} C_{R, i}$ where the added term $A_{j} x_{i j} C_{A, i}$ allows including any external parameter using $x_{i j}$ (and in our case $x_{i j}$ was constructed using a proxy to the flux). We were thus able to tie the effect observed by M09 to a particular type of effect: added/subtracted linear flux, and were able to improve on their correction due to this better understanding.

The SARS noise model can be generalized to include multiple and arbitrary $x_{i j}$ terms so that all known effects can be accounted for, simultaneously with the regular SysRem for unknown effects. These can be: locations on the CCD, pixel phases, Moon phase, temperature, and so on -i.e., any external parameter. 

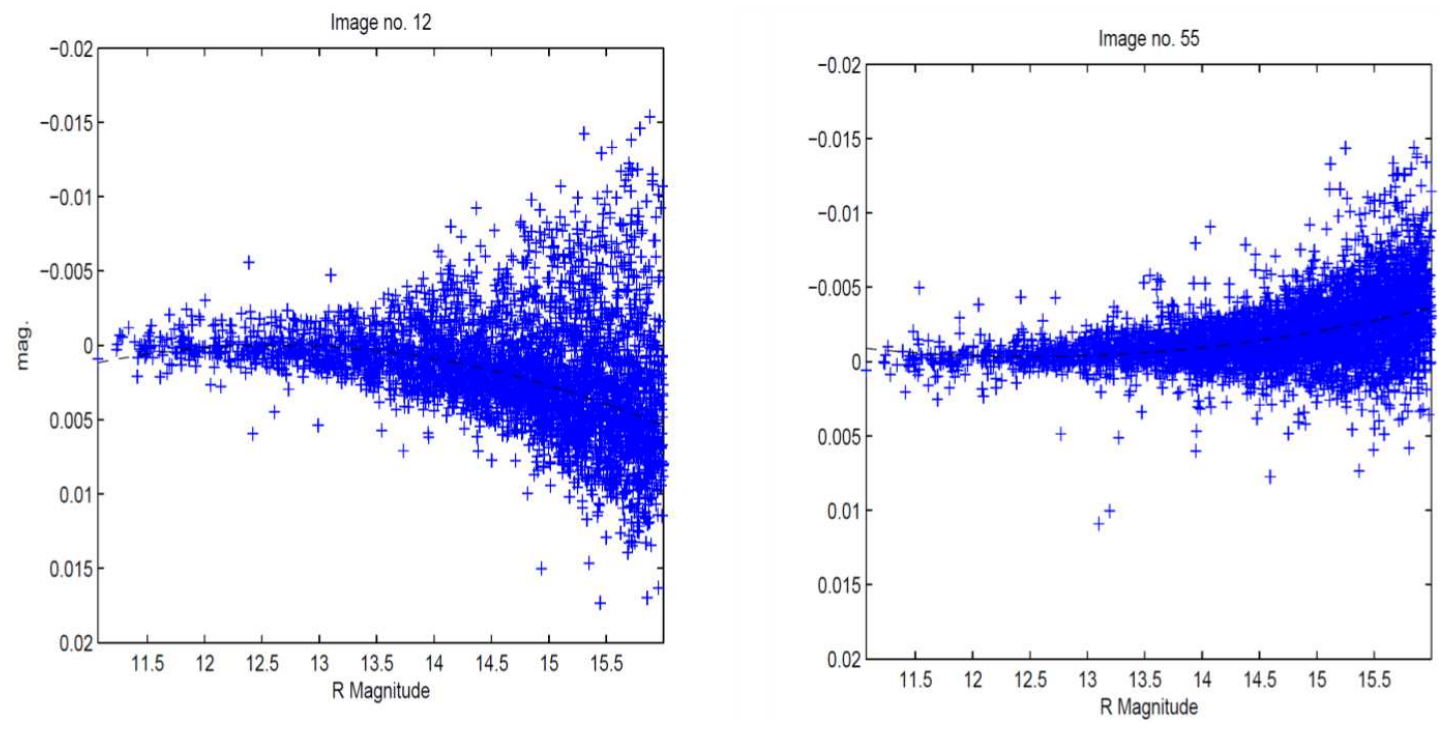

Figure 1: An extract from M09 showing the residuals of many stars in two images, vs. magnitude. These distributions should be flat, so the systematic effect discovered by M09 is obvious. Basically, the M09 correction is subtracting the parabola shown in black dashed line.

\section{Results}

As many as $80 \%$ of the SARS-processed CoRoT light curves are better corrected than after the M09 correction, and indeed about a dozen new candidates, not otherwise detected, are discovered in a typical CoRoT long run. Some of these are later discarded as unlikely planetary in nature (as identified by tests such as odd-even depth difference etc.). Still, dozens of new signals have already passed all filters and are now being followed-up as part of the normal CoRoT follow-up program. Figure 2 shows some of these signals.

Acknowledgements. The CoRoT space mission, launched on December 27th 2006, has been developed and is operated by CNES, with the contribution of Austria, Belgium, Brazil, ESA, Germany, and Spain. CoRoT data become publicly available one year after release to the Co-Is of the mission from the CoRoT archive: http://idoc-corot.ias.u-psud.fr/.

\section{References}

Mazeh, T., et al. 2009, A\&A, 506, 431

Ofir, A., et al. 2010, MNRAS, 404, L99

Tamuz, O., Mazeh, T., \& Zucker, S. 2005, MNRAS, 356, 1466 
Detection and Dynamics of Transiting Exoplanets
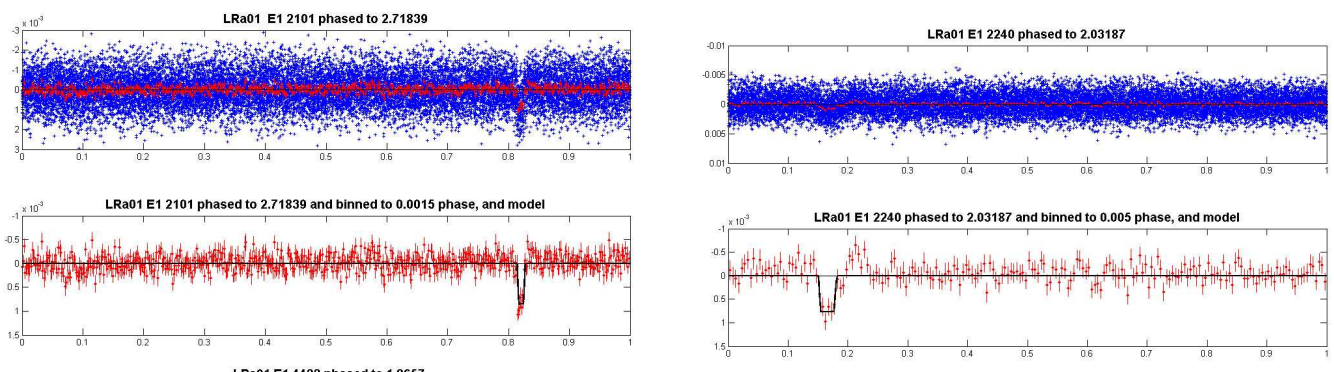

LRa01 E1 4423 phased to 1.8657
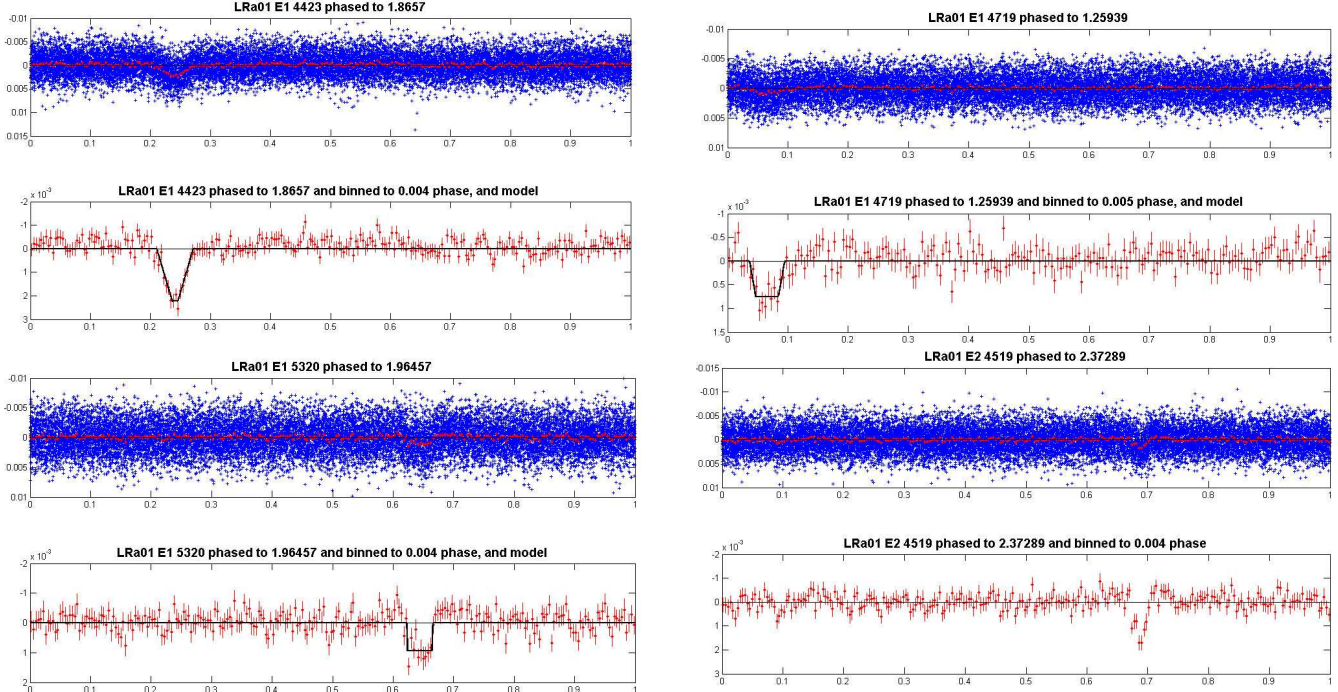

Figure 2: Six high-significance candidates (> 20 sigma detection) from a single CoRoT run (LRa01). These relatively shallow candidates (four are shallower than 1 mmag) were discovered only after SARS processing, and were later selected for follow-up. For each candidate: Top panel: phased SARS-processed original data (blue) and binned data (red). Bottom panel: binned light curve and trapezoidal model (Black). 